\title{
Folding a fish, making a mushroom: The role of diagrams in executing assembly procedures
}

\author{
LAURA R. NOVICK and DOUGLAS L. MORSE \\ Vanderbilt University, Nashville, Tennessee
}

\begin{abstract}
Three experiments examined the role of step-by-step and final-state diagrams in supporting object assembly. A total of 180 college students made origami objects from instructions consisting of text only, text plus a final-state (completed-object) diagram, or text plus step-by-step and final-state diagrams. In Experiments 1 and 2, construction accuracy in the final-diagram condition was comparable to that in the step-by-step condition when the objects required few assembly steps, but it was comparable to that in the text-only condition when many steps were required. Experiment 3 independently manipulated the number of assembly steps and the ease of seeing the steps in, or inferring them from, the final diagram. The results indicated that the ease of extracting the steps from the final diagram was the primary causal variable in the interaction with instructional condition. We interpret these results in terms of mental model construction and working memory load.
\end{abstract}

Imagine the following situation: It is your son's 5 th birthday, and you have bought him his first bicycle. He opens the box with great excitement, only to discover a pile of disconnected parts rather than the bicycle he has been so eagerly anticipating. As he exclaims with worry in his voice, "What happened to my bicycle?" you feverishly search for the instructions, hoping that the text will be clear, grammatically correct, and accompanied by appropriate diagrams.

Although a lot of research supports the general claim that diagrams are important aids to comprehension and problem solving, there is relatively little work on the role of diagrams in supporting the execution of assembly instructions. This is somewhat surprising given the ubiquity of object assembly tasks in everyday life. Adults follow diagrammed instructions to assemble bicycles, bookcases, and similar items. Likewise, children of various ages follow diagrammed instructions to assemble, for example, Lego objects, model airplanes, and origami figures.

The present research has two aims: (1) to extend research on diagrams as instructional aids to the case of object assembly, and (2) to compare the utility of stepby-step and final-state diagrams in supporting object as-

The authors thank the students in the first author's spring 1996 undergraduate seminar on "Thinking with Diagrams" for their help with all phases of Experiment 1: Amber Carlston, Mark Frymire, Susan Greene, Jessica Leffler, Deborah Martin, Aynsley McWhorter, Philippa North, and Karly Randolph. We also thank Jaime Jones for her help in collecting and coding the data for Experiment 2, Kristen Wilson for her help in collecting the data for Experiment 3, Deborah Gonzales for suggesting that we collect confidence ratings for the Experiment 3 manipulation check study. Finally, we give special thanks to Philippa North for her help with Experiment 1 as part of the class project, with all phases of Experiment 2, and with creating materials for Experiment 3. Correspondence should be addressed to L. R. Novick, Department of Psychology and Human Development, Vanderbilt University, Box 512 Peabody, Nashville, TN 37203 (e-mail: laura.novick@vanderbilt.edu). sembly. We hypothesize that final-state diagrams are primarily helpful when it is easy to see or infer the assembly steps in the diagram. In those situations, a final-state diagram can substitute for a large number of step-by-step diagrams. We discuss our findings from the perspectives of mental model construction and working memory load.

\section{Instructional Diagrams Facilitate the Construction and Use of Mental Models}

On the basis of an analysis of a wide variety of different types of diagrams from many different content domains, Cheng (1996) proposed 12 functional roles for diagrams. Two of these roles are relevant to the present discussion: Some diagrams show how an object is assembled from its component parts, and some diagrams depict the temporal order of a process by illustrating states and changes to states. The latter functional role, especially, may help reasoners generate a mental model of the process being depicted (Cheng, 1996), because the relevant information is immediately apparent in the diagrams, whereas it would have to be computed from the corresponding written description (Larkin \& Simon, 1987).

The results of several studies support Cheng's (1996) claim that diagrams facilitate the construction and use of mental models for comprehension and reasoning. Bauer and Johnson-Laird (1993) found that people were better able to reason about complex disjunctions when the problems were presented diagrammatically rather than verbally. Glenberg and Langston (1992) found improved comprehension of a four-step procedure when the text was accompanied by a diagram. Mayer, Bove, Bryman, Mars, and Tapangco (1996) found that a sequence of five annotated diagrams was better for teaching students about lightning than was a 600 -word passage.

The research on comprehension of mechanical systems is particularly relevant to our interest in assembly instructions. In both cases, the diagrams provide information 
about the relative locations of the parts of the object in question. In addition, in both cases, the information provided must enable reasoners to see or infer state changes over time. In assembly tasks, the object changes shape as it is constructed through the execution of a specific sequence of steps. Mechanical systems (e.g., pulley systems, brakes) typically have moving parts, so it is necessary to understand and be able to reason about the interactions among the parts as they move. The results of several studies support Cheng's (1996) proposed functional roles by highlighting the importance of depicting the temporal order of a process or the state changes that result as a mechanical system moves.

Hegarty and Just (1993) found that adding a diagram of a pulley system to an instructional text provided a concrete referent that reduced the load on working memory and helped learners animate their mental models. This in turn helped them understand the kinematics of the system. Mayer and Gallini (1990) found that students who received a text accompanied by a diagram that illustrated both the components and the major motions of the (brake, pump, or electric generator) system performed better on conceptual recall and problem-solving questions than did students who received the text alone or accompanied by a more impoverished diagram.

Surprisingly, we could find only a single article concerning object assembly from text and diagrams. Marcus, Cooper, and Sweller (1996) examined fifth- and sixth-graders' ability to connect resistors in series or parallel based on either written or diagrammatic instructions. All the problems required subjects to make four connections, but they differed in the number of possible connection points that had to be considered at each step in the instructions. In particular, the parallel problem required subjects to consider more possible connection points between resistors than did the series problems. Marcus et al. used Sweller's cognitive load theory to predict that the parallel problem would be more difficult than the series problems in the text condition, due to the increased cognitive load associated with that problem. In contrast, there should be little or no problem type effect in the diagrams condition. This is because the diagrams should reduce the cognitive load associated with the assembly task, because all the possible connection points are shown in the diagram and therefore need not be held in working memory. These predictions were supported in three experiments.

\section{Final-State Versus Step-by-Step Diagrams}

Although the results of Marcus et al. (1996) are important in demonstrating a benefit of diagrammatic presentation over text presentation for an assembly task, they raise an additional important issue that has not been addressed. The diagrams Marcus et al. used served Cheng's (1996) function of showing how the object was assembled from its component parts. That is, each diagram showed the final state of the object when assembled correctly. We will henceforth refer to such diagrams as final diagrams. A particular order for assembling the parts was given in the text instructions, but subjects who received the diagrams only were free to assemble the parts in any order. Partly because of the nature of the object being assembled, and partly because of the small number of steps involved, the order in which the connections were made did not affect the outcome, and, therefore, final diagrams were appropriate. In the more typical case, however, the order of assembly is important, and, therefore, it may be important that the instructional diagrams serve Cheng's function of depicting temporal order by showing states and changes to states. We will refer to such diagrams as step-by-step diagrams. The primary goal of the experiments reported here is to compare the effectiveness of step-by-step and final diagrams in aiding object assembly in the more typical situation in which the order of executing the steps is important.

\section{The Range of Object Assembly Tasks}

The category of object assembly tasks is quite broad, including, for example, the following members: building a house, assembling a bicycle, building a castle out of wooden blocks, soldering together a circuit board, assembling a jigsaw puzzle, building a sand castle, constructing a three-dimensional cardboard model of a building, and folding objects out of paper. Several distinctions or dimensions seem to characterize the diversity in this domain. We discuss these dimensions in terms of their extremes, although, clearly, intermediate cases are possible as well. There may be few or many solution paths possible (e.g., assembling a bookcase vs. building a house or assembling a jigsaw puzzle). Some objects are constructed by connecting many separate, already-assembled components, whereas other objects emerge from a sequence of transformations applied to a single component (e.g., building a bicycle vs. folding a paper airplane). In some domains, the basic components are highly differentiated, whereas, in other domains, they are less easily differentiated (e.g., assembling exercise equipment vs. soldering together a circuit board or building a sand castle).

We chose origami, a Japanese paper-folding task enjoyed by both children and adults, as our domain of application. In this domain, there is generally only a single possible solution path, and the object emerges as the result of a sequence of transformations applied to a single, undifferentiated component (i.e., a square piece of paper). There are a finite number of basic operations (i.e., transformations of the paper). Although these operations typically are conceived of as isolated steps during the construction process, certain steps do sometimes aggregate to form higher order units of meaning that recur across (or even within) objects.

The task of origami has several advantages. First, the order in which the folds are made is crucial for correctly constructing the object. This is an important characteristic of most (although not all) object assembly tasks. Second, origami objects vary widely in difficulty. Thus, it is possible to determine whether the effectiveness of 
different types of diagrams depends on the difficulty of the assembly task. Third, most adults have some familiarity with this domain-almost everyone has folded paper airplanes!--although they are not highly skilled. Mayer and Gallini's (1990) results suggest that less knowledgeable subjects may be more sensitive to the format in which information is presented (but see Hegarty \& Just, 1993, for opposing results). Fourth, most people find the task to be enjoyable and challenging. They are generally motivated to do well, and they take pride in their accomplishment when they successfully construct an object.

\section{Overview of the Experiments and Predictions}

In Experiments 1 and 2, we manipulated the format of the instructions and the number of steps required to create the origami objects. At the low end, the number of steps was only slightly greater than that required for Marcus et al.'s (1996) task (about 7). At the high end, the objects required approximately three times as many steps (about 23). The objects requiring more steps also required a greater variety of and more difficult manipulations of the paper for successful construction. Subjects received instructions in one of three formats: (1) text only, (2) text accompanied by a final diagram, and (3) text accompanied by both step-by-step and final diagrams. Our main dependent measure was the accuracy with which the objects were constructed.

In the text-only condition, builders must create a mental model of the operations described in the text and then map those manipulations onto the actual object under construction. Both the model construction and mapping processes are likely to be error-prone. Moreover, it is difficult to recover from any errors made, because they are likely to remain undetected unless they make it impossible to execute a subsequent step. Even then, the feedback does not indicate the point at which the error was made.

In contrast, in the step-by-step condition, the model construction process is greatly simplified for all steps accompanied by diagrams (which for our materials was almost every step), and the mapping process is facilitated because both the model and the object are perceptually available. Moreover, the diagram showing how to execute step $n$ typically illustrates what the object should look like after having completed step $n-1$. This feedback enables builders in the step-by-step condition to detect and recover from errors. Therefore, we predict that accuracy will be higher in the step-by-step condition than in the text-only condition.

The predictions for the final-diagram condition are more complicated and more interesting. We predict that the effectiveness of a final diagram for supporting object assembly depends, to a first approximation, on the difficulty of the object assembly task. Difficulty in the domain of origami is a multifaceted construct. In part, it depends on the number of steps to be executed, with more steps generally indicating greater difficulty. In part, it depends on the difficulty of the required manipulations. Some manipulations are fairly easy (e.g., fold the paper in half, turn the object over), whereas others are more difficult (e.g., use one hand to separate two layers of paper while simultaneously using the other hand to crease down another part of the object).

When there are few steps and the required manipulations are fairly easy, many or most of the steps are likely to be visible in or easily inferable from the final diagram. This should allow builders to "index" the written descriptions of the procedures to parts of the final diagram (Glenberg \& Robertson, 1999). That is, builders should be able to establish a reference relation between a part of a written step (e.g., "fold the keyboard of the piano up") and the appropriate part of the diagram (the keyboard in the depicted piano). But, when there are many steps and the manipulations are more complex, few of the steps are likely to be visible in or inferable from the final diagram. For example, later manipulations may obscure important landmarks from earlier ones. In this case, it will not be possible to index the written descriptions to parts of the diagram, and the errors inherent in relying on a constantly changing mental model are likely to compound. This analysis led us to predict an interaction between number of steps and instructional condition in Experiments 1 and 2 , such that performance in the final-diagram condition would be similar to that in the step-by-step condition when there are few steps but like that in the text-only condition when there are many steps.

Like Bauer and Johnson-Laird (1993), we believe that diagrams are beneficial when they facilitate visualizing possible states of the world and conceptualizing transitions between states. And, like Larkin and Simon (1987), we believe that the effectiveness of diagrams for reasoning and instruction depends on the ease of extracting information from them that is only implicit. Applying these principles to object assembly tasks, such as origami, we contend that final diagrams are helpful to the extent that information about the individual assembly steps can be easily extracted from them. Objects that require few steps and for which the manipulations of the paper are straightforward are likely to be ones for which the steps are easily visible in or inferable from the final diagramhence our predictions for Experiments 1 and 2. We maintain, however, that the number of steps and the difficulty of the manipulations are not the only important causal variables. Rather, we argue that the ease of extracting the steps from the final diagram plays at least an equal, if not more important, role.

Figure 1 illustrates how final diagrams for origami objects can differ in the degree to which the individual assembly steps are evident. Sixteen steps (i.e., folds or other manipulations of the paper) are required to make both the piano and the mushroom. The first seven steps for each object are described in the figure. As shown in the left panel, these steps are fairly easy to extract from the final diagram for the piano. In contrast, as shown in the right panel, these early steps are not readily apparent in the final diagram for the mushroom. 


\section{Piano: \\ Easier to Extract the Steps from the Final Diagram}

1. With the white side facing up, place the piece of paper so that it is in the shape of a square. Fold the paper in half by bringing the top side down to the bottom side, forming a rectangle.

2. Fold the paper in half again by bringing the right side over to the left side, forming a square. Undo this fold so you have formed a crease down the middle of the rectangle.

3. Fold the left edge of the figure toward the middle so that it lines up along the center crease. Repeat on the right side of the figure.

4. Slip the pointing finger of your left hand between the two layers of the left flap you made in step 3 (the paper is colored white between the two layers). Gently press down with this finger to hold the bottom layer of the left flap in place. Then put the pointing finger of your right hand on the very tip of the upper right comer of the left flap. Gently push in on this comer tip so that the left flap starts to open up. Continue to move this corner tip left and down so that the left flap opens up completely and lies flat. This will form a colored triangle centered over a white rectangle. Crease the triangle into place.

5. Repeat this step on the flap at the right side of the figure.

$$
\text { : }
$$

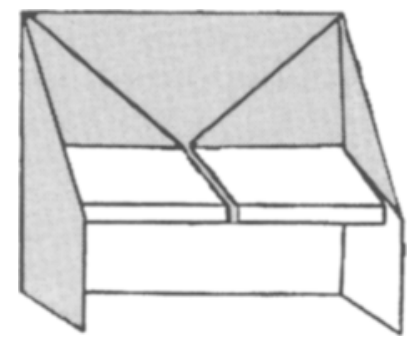

\section{Mushroom: Harder to Extract the Steps from the Final Diagram}

1. With the white side facing up, place the piece of paper so that it is in the shape of a diamond. Fold the paper in half by bringing the bottom point up to the top point, forming a triangle. Undo this fold so you have formed a crease across the middle of the diamond.

2. Fold the paper in half again by bringing the right side over to the left side, forming a triangle. Undo this fold so you have formed a crease down the middle of the diamond.

3. Fold the top point of the diamond down to the center of the paper, where the two creases intersect.

4. Repeat the fold from step 3 on the left and right points of the diamond, again folding to where the two creases intersect.

:

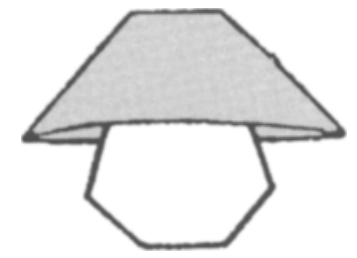

Figure 1. Partial text instructions and final diagrams for the 16-step objects used in Experiment 3. Note that it is easier to extract the individual assembly steps from the final diagram for the piano (left panel) than for the mushroom (right panel). Note-The piano final diagram is from Easy Origami (p. 21), by John Montroll, 1992, New York: Dover Publications. Copyright 1992 by John Montroll. The mushroom final diagram is from Origami Made Easy (p. 75), by Kunihiko Kasahara, 1973, Elmsford, NY: Japan Publications, Inc. Copyright 1973 by Kunihiko Kasahara. Both diagrams adapted with permission of the respective copyright holders.

In Experiment 3, we directly test our hypothesis that the ease of mentally extracting the steps from the final diagram is an important determiner of performance in the final-diagram condition. We predict that when the steps are easy to see in or infer from the final diagram, performance should be comparable in the final-diagram and step-by-step conditions. But when the steps are difficult to extract from the final diagram, performance in the final-diagram condition should be considerably worse than that in the step-by-step condition. These predictions are consistent with Marcus et al.'s (1996) general claim that difficulty in understanding depends not so much on the amount of information that must be assimilated but on the amount of information that must be held in working memory simultaneously. When the steps are difficult to extract from the final diagram, a greater number of alternative models must be held in working memory, and step-by-step diagrams are likely to be required for accurate object assembly. But when the steps are easy to extract from the final diagram, the load on working memory is reduced, and the number of diagrams used to illustrate the written instructions should matter less.

\section{EXPERIMENT 1}

\section{Method}

Subjects. The subjects were 72 Vanderbilt University undergraduates (41 females, 31 males), who participated viluntarily at the request of the experimenters. The experimenters $w$ :re eight students enrolled in a cognitive psychology seminar.

We created a short questionnaire to assess the subjects' prior paper-folding experience. The two questions asked: "How much experience have you had in the past making different kinds of paper airplanes [origami objects]?" The five response options for each question were: (1) a lot; considerably more than most people I know; 
(2) a fair amount; probably somewhat more than most people; (3) some; (4) only a little; (5) I have never made paper airplanes [origami objects] before. We scored the subjects' responses on a 0-4 scale, with higher numbers indicating greater experience. The mean paper airplane score was $2.47(S D=0.87)$, and the mean origami score was $1.78(S D=0.83)$. As expected, our subjects were not highly experienced in the origami domain.

Design. There were two independent variables. Instructional condition was manipulated between subjects. In the text-only condition, the subjects received a written description of how to fold the origami objects. In the final-diagram condition, these instructions were accompanied, at the end, by a diagram depicting the completed object. In the step-by-step condition, the written instructions were accompanied by intermediate diagrams for most of the numbered procedures, which illustrated the manipulations described in the corresponding text. A finished-product illustration also appeared at the end of the instructions. The subjects were randomly assigned to these conditions ( 24 per condition), which were equally distributed across the eight experimenters.

The second independent variable, number of steps, was manipulated within subjects. The dog required 5 steps, and the boat required 16 steps. Each manipulation of the paper, usually a fold, was counted as a single step. In the written instructions, some numbered procedures actually involved several manipulations of the paper. For example, for the piano and mushroom shown in Figure 1, four or five numbered procedures include seven manipulations (i.e., 7 steps).

Materials. Both objects came from an origami book by Sakade (1958). All of the steps for the dog were simple folds. Most of the steps for the boat were simple folds, but the last step was more complicated, as it involved turning the object inside out. The text portions of the instructions were rewritten, most notably by substituting verbal descriptions for direct references to the diagrams. This was done so that the text would be comprehensible in the absence of diagrams (see Figure 1). By comprehensible, we mean that a motivated person, working slowly and carefully, would be able to construct the object from the written instructions, because the text accurately described the required manipulations of the paper. This was verified in pilot work. The diagrams from the original instructions were scanned into a computer file, cleaned up, and inserted at appropriate places in the text for the final-diagram and step-by-step conditions.

Origami objects usually are created using special thin, square pieces of paper made for that purpose. Two different sizes of origami paper were used: $4.5 \times 4.5$ in. $(11.4 \times 11.4 \mathrm{~cm})$ for the $\mathrm{dog}$, and $9 \times 9$ in. $(22.9 \times 22.9 \mathrm{~cm})$ for the boat.

Procedure. The subjects participated individually in a single session. First, they completed the prior experience questionnaire. Then, they were told what type of origami instructions they would receive, as appropriate to the instructional condition to which they had been assigned. They were also told that the objects would increase in difficulty across the experiment. The subjects were then given a small piece of origami paper and instructions for making the dog. They were allowed up to $10 \mathrm{~min}$ to make this object. All subjects finished before that time, and the experimenter recorded the amount of time taken. The subjects were then given a large piece of paper and instructions for making the boat. They had up to $15 \mathrm{~min}$ to work on this object. All but $8(11 \%)$ subjects finished the boat, and the experimenter recorded the time taken. For the 8 who did not finish, a time of $15 \mathrm{~min}$ was assigned and the partially constructed boat was collected.

\section{Results and Discussion}

Accuracy. The experimenters, blind to condition, coded assembly accuracy for each object on a $0-4$ scale. ${ }^{2}$ A score of 0 was given if there were major errors very early in the construction process and the object that was turned in was completely wrong. A score of 1 or 2 was given if the object was partially completed, with 1 representing only a little progress and 2 representing good progress. Operational definitions of "little progress" and "good progress" were identified for each object that made sense in the context of what was required for successful construction of that object. A score of 3 was given if the object was essentially correct, with only a minor error in the construction process. A score of 4 denoted a completely successful construction attempt.

These scores were analyzed with a $3 \times 2$ analysis of variance (ANOVA) with instructional condition as a grouping factor and number of steps as a repeated measure. We made three predictions: (1) accuracy should decrease with increasing number of steps, (2) accuracy should be higher in the step-by-step condition than in the text-only condition, and (3) performance in the finaldiagram condition should depend on the number of assembly steps required. That is, we predicted an interaction between instructional condition and number of steps. These predictions were supported.

There was a main effect of number of steps, with means of 3.53 and 3.22 for the 5- and 16-step objects, respectively $\left[F(1,69)=7.28, M S_{\mathrm{e}}=0.4620, p<.01\right]$. A greater number of steps means additional opportunity for error, so this finding makes sense. Accuracy also varied across conditions, with means of $3.19,3.21$, and 3.73 for the textonly, final-diagram, and step-by-step conditions, respectively $\left[F(2,69)=2.98, M S_{\mathrm{e}}=1.518, p<.06\right]$. As we predicted, having many diagrams (step-by-step condition) was better than having no diagrams at all (textonly condition).

Of more interest was the condition $\times$ number of steps interaction $\left[F(2,69)=7.32, M S_{\mathrm{e}}=0.4620, p<.01\right]$. As shown in Figure 2, performance in the final-diagram condition was similar to that in the step-by-step condition for the 5-step object, but it was similar to that in the text-only condition for the 16-step object. Although the graph shows that performance was numerically worse in the final-diagram condition than in the text-only condition for the 16-step object, this is not a reliable difference $[t(46)=1.17, S E=0.3557, p>.24]$.

We interpret the interaction shown in Figure 2 in terms of mental model construction and working memory load. It should be relatively easy to mentally extract the individual assembly steps from the final diagram for our 5step dog. This should enable the subjects to confirm or revise their mental model of the assembly steps derived from the written instructions; also, it should help them map the required manipulations onto the object in front of them, due to the visual similarity between parts of the object and parts of the final diagram. Thus, the step-bystep diagrams are largely superfluous. But it should be more difficult to extract the steps from the final diagram for the 16-step boat, because of the greater discrepancy between the start state (a flat square of paper) and the final 

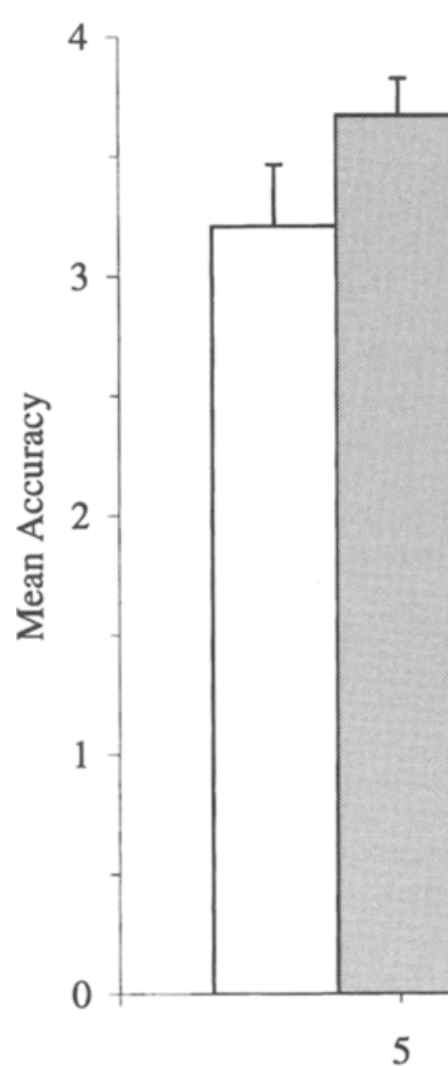

$\square$ Text Only
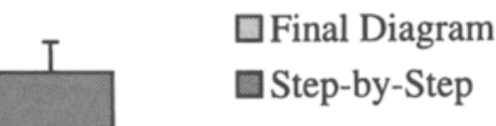

.

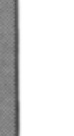

\section{Number of Steps}

Figure 2. Mean accuracy $(+S E)$ as a function of number of steps for the text-only, final-diagram, and step-by-step conditions in Experiment 1 ( $n=24$ per condition).

state. Hence, the step-by-step diagrams are needed for checking one's comprehension of the text and for mapping that understanding onto the under-construction boat.

Methodological issues. There are two weaknesses with this experiment. First, because we had only one object for each number of steps, both the interaction and the main effect of number of steps may be specific to the objects we selected. Second, because some subjects gave up before the end of the allotted time, we were unable to measure the subjects' efficiency in constructing the objects. We presume that people need more time to complete tasks having more steps; however, they might work more efficiently (e.g., require less time per step) when they have more diagrams, and such a benefit might interact with the number of steps involved. We were unable to consider this possibility because we could not compute an appropriate measure. Experiment 2 was designed to overcome these potential problems, while otherwise replicating Experiment 1.

\section{EXPERIMENT 2}

The materials and procedure of Experiment 1 were changed in three ways for this experiment. First, we in- creased the difference in the number of assembly steps between the few-step objects and the many-step objects. Second, for each level of number of steps, we used three objects instead of one. Finally, we had subjects record how much time they spent constructing each object, and we simultaneously tracked the number of steps they attempted to complete. This enabled us to examine subjects' efficiency in the construction process by computing the (average) amount of time that it took to complete each step.

\section{Method}

Subjects. The subjects were 60 Vanderbilt University undergraduates ( 30 females, 30 males) who participated in partial fulfillment of course requirements. Their mean paper airplane experience score was $1.53(S D=0.70)$, and their mean origami experience score was $0.92(S D=0.89)$. This average origami score indicates that the subjects had "only a little" prior experience.

Design. We again used a $3 \times 2$ factorial design with instructional condition (text-only, final-diagram, or step-by-step) as a grouping factor and number of steps (few or many) as a within-subjects factor. Twenty subjects were randomly assigned to each of the instructional conditions, which were equally distributed across two experimenters. On average, the three few-step objects required 7 steps (range of 5-10), and the three many-step objects required 23 steps (range of 18-31). We classified objects as having either few or many steps because, in origami, as in many object assembly do- 
mains, not all steps are equivalent in difficulty. The difficulty of constructing an object will depend partly on the number of steps required and partly on the difficulty of executing those steps. If there is only a small difference in the number of steps required for two objects, then the one with fewer steps could easily be harder to construct than the one with more steps if those fewer steps are more difficult. Thus, the design decisions we made serve to provide a robust manipulation of the number of steps factor.

Materials. Two objects - the dog ( 5 steps) and the boat ( 18 steps) - were the same as those used in Experiment 1. However, we added two steps (a fold and corresponding unfold) to the written instructions for the boat to form a crease line down the middle of the paper. The crease was a useful landmark for the subjects so they would not have to make a subjective judgment as to the location of the middle of the paper, which was necessary for making the subsequent fold. Making creases is an important part of the assembly procedure for many origami objects. To supplement the dog and the boat, we selected a 6-step whale (Montroll, 1992), a 10-step penguin (Temko, 1986), a 21-step bird (Froebel-Kan Co., 1991), and a 31 -step fish (Neale \& Hull, 1994). These six objects are representative of origami objects requiring these numbers of steps.

The few- and many-step objects also differed in the variety and complexity of the folds required for construction. The few-step objects generally required folds that fell along edges or crease lines, as well as other simple manipulations of the paper (e.g., turning the object over). The many-step objects, on the other hand, had some folds that did not fall along edges or crease lines; and they sometimes required more complicated manipulations of the paper, such as opening pockets, tucking flaps underneath parts of the object, or turning the object inside-out.

As in Experiment 1, the text portions of the instructions were rewritten so that they would be comprehensible in the absence of diagrams. The diagrams were created as in Experiment 1. For the final-diagram and step-by-step conditions, the final diagrams were glued to $5 \times 8$ in. $(12.7 \times 20.3 \mathrm{~cm})$ note cards and presented along with the rest of the instructions. Thus, in these conditions, the final diagram was readily available throughout the construction process. The subjects used $7 \times 7$ in. $(17.8 \times 17.8 \mathrm{~cm})$ origami paper to make all of the objects.

Procedure. The subjects began the experiment by completing the questionnaire. Then, they constructed the three few-step objects, followed by the three many-step objects. The ordering of the objects within each set was counterbalanced such that each object appeared once in the first, second, and third positions (within the set). Before attempting to construct each object, the subjects started a timer. When they finished, they stopped the timer and recorded the amount of time they spent constructing the object. The subjects were told that they could work as long as they needed on each object, but that if they took too long, the experimenter would stop them and let them go on to the next object so that they would have a chance to attempt each object. The experimenter interrupted the subjects only if they took longer than $25 \mathrm{~min}$ on one of the many-step objects. One and a half hours was allocated for the experiment, and all subjects finished within that amount of time.

Unbeknownst to the subjects, the experimenter monitored their progress on each object and noted the completion of each numbered procedure in the text. A procedure was deemed completed if the subject attempted one or more manipulations of the paper corresponding to that procedure, regardless of whether the manipulations were executed correctly. The subjects attempted an average of $96 \%$ ( $S D=12 \%$ ) of the steps, with a range of $90 \%-99 \%$ across the six cells in the design.

\section{Results and Discussion}

Accuracy. Accuracy for each object was coded on a $0 / 1$ scale. A score of 1 denoted a successful construction attempt; otherwise, a score of 0 was given. Finer grained distinctions were not necessary because the subjects almost always attempted all the steps. Thus, there was not much variability in the quality of the incorrect object constructions. Almost all would have received considerable partial credit using the Experiment 1 coding scheme. For each subject, a mean accuracy score was computed for the three few-step objects and the three many-step objects.

These scores were analyzed with a $3 \times 2$ ANOVA with instructional condition as a grouping factor and number of steps as a repeated measure. We predicted that (1) accuracy would decrease with increasing number of steps, (2) accuracy would be higher in the step-by-step condition than in the text-only condition, and (3) instructional condition and number of steps would interact such that accuracy in the final-diagram condition would be like that in the step-by-step condition for the few-step objects but like that in the text-only condition for the many-step objects.

As shown in Figure 3, all three predictions were supported. Accuracy decreased from .76 to .46 with increasing number of steps $\left[F(1,57)=51.21, M S_{e}=0.0527\right.$, $p<.001]$. Accuracy also varied reliably across conditions, with means of $.46, .57$, and .79 for the text-only, final-diagram, and step-by-step conditions, respectively $\left[F(2,57)=8.97, M S_{\mathrm{e}}=0.1290, p<.001\right]$. The interaction was also reliable $\left[F(2,57)=3.85, M S_{\mathrm{e}}=0.0527\right.$, $p<.03]$. The mean difference between the step-by-step and final-diagram conditions was .09 for the few-step objects but .37 for the many-step objects. In contrast, the mean difference between the text-only and final-diagram conditions showed the opposite pattern: .16 and .05 for the few-step and many-step objects, respectively. For the 5- to 10-step objects, having just the final diagram was almost as good as having intermediate diagrams for most steps in addition to the final diagram. But for the 18- to 31 -step objects, adding the final diagram to the text instructions conferred no benefit.

As discussed in the introduction, we suspect that as more steps are required, it becomes increasingly difficult to extract the individual assembly steps from the final diagram. This in turn makes it harder to construct an accurate mental model of the construction step at hand and to map the required manipulation onto the partially constructed object, which thus increases the probability of error. These errors are likely to compound throughout the object assembly task. We hypothesize that the ease of seeing the steps in, or inferring them from, the final diagram is a more important explanatory variable than the number of steps to be completed. Although it seems likely that these two factors are naturally correlated in the origami domain, and probably in other assembly domains as well, it should be possible to unconfound them experimentally. We do this in Experiment 3.

Construction time. To measure the subjects' efficiency at constructing the objects, we computed a time per step score for each object by dividing the total time spent constructing the object by the number of steps com- 


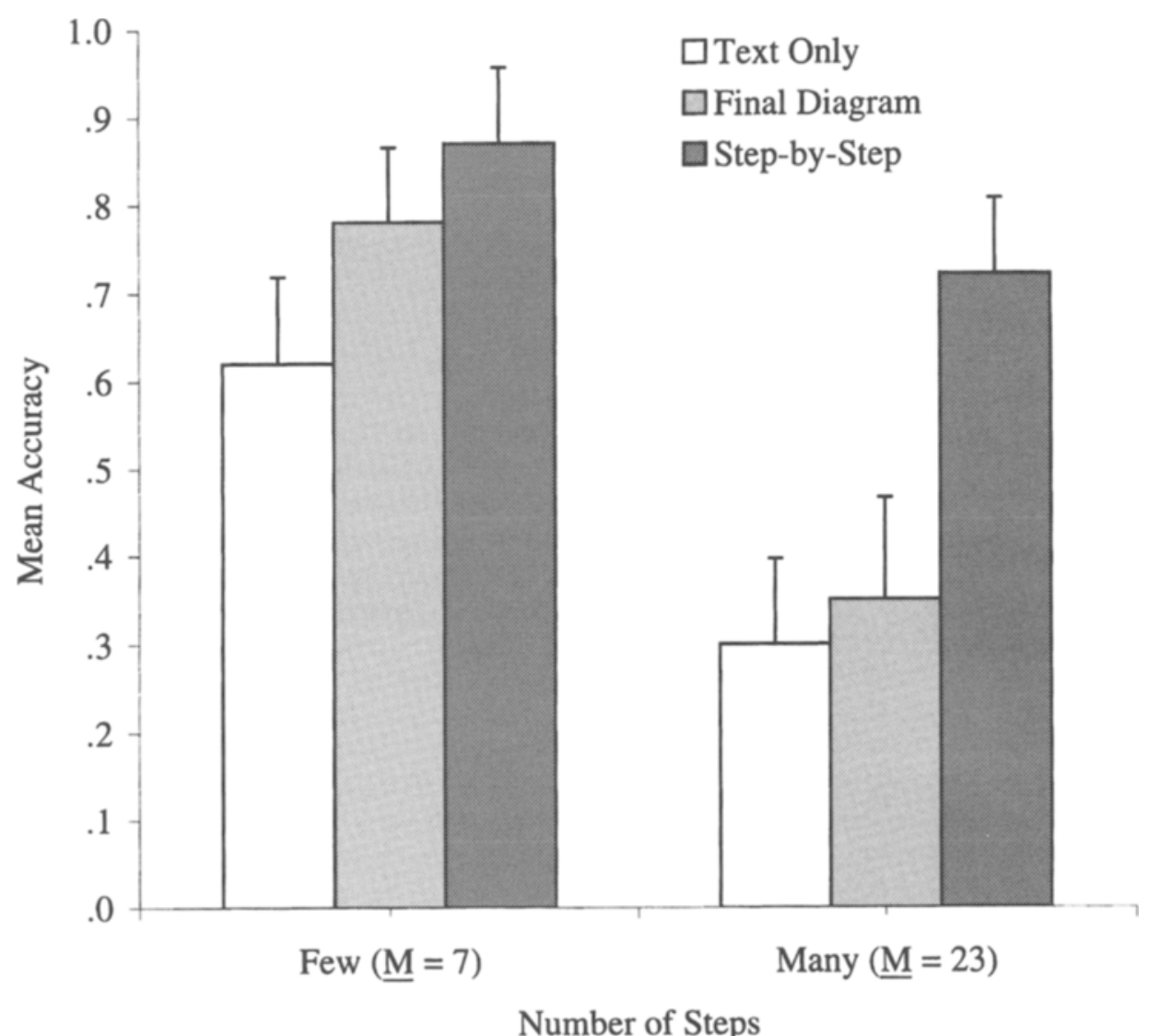

Figure 3. Mean accuracy ( $+S E$ ) as a function of number of steps for the text-only, final-diagram, and step-by-step conditions in Experiment 2 ( $n=20$ per condition).

pleted (assessed on line by the experimenter). This measure is a more useful indicator than is raw time of how difficult the subjects found the assembly task because (1) it is not appropriate to compare the raw times of subjects who completed all the steps with the times of subjects who gave up before the last step and (2) it obviously takes more time to do more things, and, hence, raw times naturally increase as the number of steps increases. For each subject, a mean time per step score was computed for the three few-step and three many-step objects.

A $3 \times 2$ ANOVA with instructional condition as a grouping factor and number of steps as a repeated measure indicated that the time per step increased from 0.46 to $0.55 \mathrm{~min}$ per step for the few-step and many-step objects, respectively $\left[F(1,57)=14.70, M S_{\mathrm{e}}=0.0167, p<\right.$ $.001]$. This difference may have been due to the fact that the many-step objects required more varied and complicated manipulations of the paper. Time per step also differed across instructional conditions, with means of $0.60,0.53$, and 0.37 for the text-only, final-diagram, and step-by-step conditions, respectively $[F(2,57)=13.65$, $\left.M S_{\mathrm{e}}=0.0409, p<.001\right]$. The step-by-step diagrams enhanced the subjects' efficiency by $35 \%$ over the textonly and final-diagram conditions. The instructional condition $\times$ number of steps interaction was not reliable $\left[F(2,57)<1, M S_{\mathrm{e}}=0.0167\right]$. We will discuss these results in conjunction with presenting the Experiment 3 construction time data.

\section{EXPERIMENT 3}

Experiment 3 was designed to directly test our hypothesis that the ease of seeing the assembly steps in, or inferring them from, a finished object diagram is the primary causal factor underlying the effectiveness of a final diagram in aiding construction accuracy. Because the critical comparison in terms of testing this hypothesis is between the step-by-step condition, in which it is always easy to extract the steps, and the final-diagram condition, in which we hypothesize that the ease of seeing (or inferring) the steps varies, only these conditions were used. Ease of extracting the steps from the final diagram and number of steps were manipulated independently.

If our hypothesis is correct, we should find an interaction between instructional condition and ease of extracting the steps from the final diagram: When the steps are easy to discern in the final diagram, construction accuracy should be similar in the step-by-step and final- 
diagram conditions. But when it is difficult to extract the steps, accuracy should be lower in the final-diagram condition. Thus, we predict that the ease of extracting the steps from the final diagram will have a larger effect on accuracy in the final-diagram condition than in the stepby-step condition. This prediction hinges on our assumption that, in the final-diagram condition, subjects use the final diagram to help them construct and update a mental model of the assembly task.

Another possibility is that subjects use the final diagram only as a goal state against which to check their performance at the end of the assembly task. Because all the procedural information is in the written instructions, subjects use only those instructions to guide their construction. According to this view, all final diagrams are created equal. ${ }^{3}$ Thus, this alternative account of the role of final diagrams in object assembly predicts that subjects in the final-diagram condition should perform equally well regardless of the ease of extracting the steps from the final diagram.

\section{Method}

Subjects. The subjects were 48 Vanderbilt University undergraduates ( 27 females, 21 males) who participated in partial fulfillment of course requirements. Their mean paper airplane experience score was $1.40(S D=0.79)$, and their mean origami experience score was $0.98(S D=0.98)$. This average origami score indicates that the subjects had "only a little" prior experience.

Design. This experiment employed a $2 \times 3 \times 2$ factorial design with instructional condition (final-diagram vs. step-by-step) as a grouping factor and number of steps $(10,13$, or 16) and ease of extracting the steps from the final diagram (easier or harder) as withinsubjects factors. Twenty-four subjects were randomly assigned to each instructional condition.

We initially classified each origami object on the basis of whether it seemed to us that it was easier or harder to see or infer the assembly steps in the final diagram. Because our initial assignment of objects to levels of this manipulation was based on our intuitions, which may differ from those of our subjects due to our greater expertise in this domain, we also conducted a manipulation check study. This study will be described in the Results section.

Materials. The whale (6 steps) from Experiment 2 was used as a practice object. Six new objects were selected in an attempt to instantiate the ease of extracting the steps manipulation at each of the three levels of the number of steps factor. For the 10-step objects, we used a penguin and a cat from Montroll (1992). The penguin was similar to the one used in Experiment 2 with respect to final appearance and the types of folds required for construction. However, it had a different ordering of the steps, which we thought might make it easier to extract the steps from the final diagram. The last step for the cat involved turning the object over. Thus, most of the intermediate folds seemed difficult to extract from the final diagram.

For the 13-step objects, we used a shield (easier to extract the steps; Neale \& Hull, 1994) and a duck (harder to extract the steps; Temko, 1986). For the duck, a step about halfway through the instructions required the object to be folded back on itself, so that most of the earlier steps and roughly half of the middle steps were not visible as folds in the final diagram. For the 16-step objects, we used the piano (easier to extract the steps; Montroll, 1992) and mushroom (harder to extract the steps; Kasahara, 1973) shown in Figure 1. The last step of the mushroom was to turn the object over, so most of the intermediate steps were not visible in the final diagram.
The written instructions and diagrams were generated as in Experiments 1 and 2, with the final diagrams presented on note cards as in Experiment 2 . The subjects used $7 \times 7$ in. $(17.8 \times 17.8 \mathrm{~cm})$ origami paper to construct all of the objects

Procedure. The procedure followed that of Experiment 2 except that the subjects created seven objects rather than six. The first object was always the whale. It served as a practice object and was not scored, although the subjects were not told this fact. The order of the six experimental objects was counterbalanced using a balanced Latin square. Overall, the subjects attempted $98 \%(S D=8 \%)$ of the assembly steps, with a range of $95 \%-100 \%$ across all the cells in the design.

\section{Results and Discussion}

Manipulation check. Because the ease of extracting the assembly steps from the final diagram is somewhat subjective and might depend in part on one's expertise in this domain, 36 additional Vanderbilt University students ( 18 female, 18 male) participated in a manipulation check study. These subjects received both 10 -step objects, both 13-step objects, or both 16-step objects ( 12 subjects for each number of steps). The order of the two objects for each number of steps was counterbalanced. All subjects received the 6-step whale first as practice, although they were not told it was for practice. For each object, the subjects received the written instructions and the final diagram, but no origami paper. Arrows were drawn on the final diagrams to indicate every visible fold, and the subjects had to label those arrows with the step number from the written instructions that was used to create that fold. The subjects then rated their confidence in their labels on a 5-point scale, with 1 indicating not at all confident, 3 indicating somewhat confident, and 5 indicating very confident. To ensure that the subjects understood the labeling task, the experimenter demonstrated it with a 7-step example object (a hat created by the authors).

Typically, only one step number from the written instructions corresponded to each fold in the final diagrams. However, several folds were created from more than one step. In these cases, one step was designated as the primary step because it was where the greatest amount of construction occurred. For example, a flap was made in the piano across three numbered steps: a crease line was first made as a landmark, a fold was later made along that crease, and, eventually, the resulting flap was adjusted by pulling it out. The middle step-where the fold was actually made - was designated as the primary step. The subjects received one point for each correctly labeled fold and half a point for each partially correct label. For multistep folds, the primary step, but not the others, had to be listed to get full credit. A label was deemed partially correct if (1) it included both a correct and an incorrect step number or (2) it included only correct step numbers, but the primary step number was omitted. Because the objects differed in the number of folds visible in the final diagram (ranging from 5 to 14), the accuracy scores were converted to proportions. 
If subjects agree with our assessment of the ease of extracting the assembly steps from the final diagrams, they should be more accurate in labeling the folds, and more confident in their labels, for the penguin than for the cat (10 steps), for the shield than for the duck ( 13 steps), and for the piano than for the mushroom (16 steps). These results were obtained for the 13-and 16-step objects, but not for the 10-step objects. For accuracy, there was a reliable mean difference (hypothesized easier minus hypothesized harder) of .29 (.64 vs. .35) for the 13-step objects $[t(11)=2.55, S E=0.1138, p<.03]$ and $.20(.54 \mathrm{vs}$. .34 ) for the 16-step objects $[t(11)=2.55, S E=0.0777$, $p<.03$ ], but no reliable difference for the 10 -step objects $[t(11)=1.81, S E=0.0666, p>.09]$ (.56 vs. .44). For the confidence ratings, there was a reliable mean difference of 1.92 (3.58 vs. 1.67) for the 13-step objects $[t(11)=6.13, S E=0.310, p<.001]$ and $0.92(2.58 \mathrm{vs}$. 1.67) for the 16-step objects $[t(11)=4.01, S E=0.230$, $p<.003]$. Not only was the difference for the 10-step objects not reliable $[t(11)=1.00, S E=0.250, p>.33]$, but it went in the wrong direction ( 2.58 vs. 2.83 ).

To summarize, for the 13- and 16-step objects, both labeling accuracy and labeling confidence indicate that we effectively manipulated the ease of extracting the as- sembly steps from the final diagrams. For the 10 -step objects, however, the measures yielded discrepant results that are best summarized by concluding that we failed to adequately manipulate the ease of extracting the steps: For labeling accuracy, performance was marginally better for the hypothesized easier-to-extract-the-steps object. For labeling confidence, there was a small difference in the opposite direction. Given these results, it is not appropriate to include the 10-step objects in our analyses of the origami construction data. We will consider one possible source of the difficulty in instantiating this manipulation in the General Discussion. ${ }^{4}$

Accuracy. As in Experiment 2, accuracy was scored as 0 or 1 . These scores were analyzed with a $2 \times 2 \times 2$ ANOVA with instructional condition as a grouping factor and number of steps and ease of extracting the steps from the final diagram as within-subjects factors. We made four predictions concerning the results of this analysis: (1) accuracy should be greater for the easier-toextract-the-steps objects than for the harder-to-extractthe-steps objects, (2) accuracy should decrease as number of steps increases, (3) accuracy should be higher in the step-by-step condition than in the final-diagram condition, and (4) instructional condition should interact with

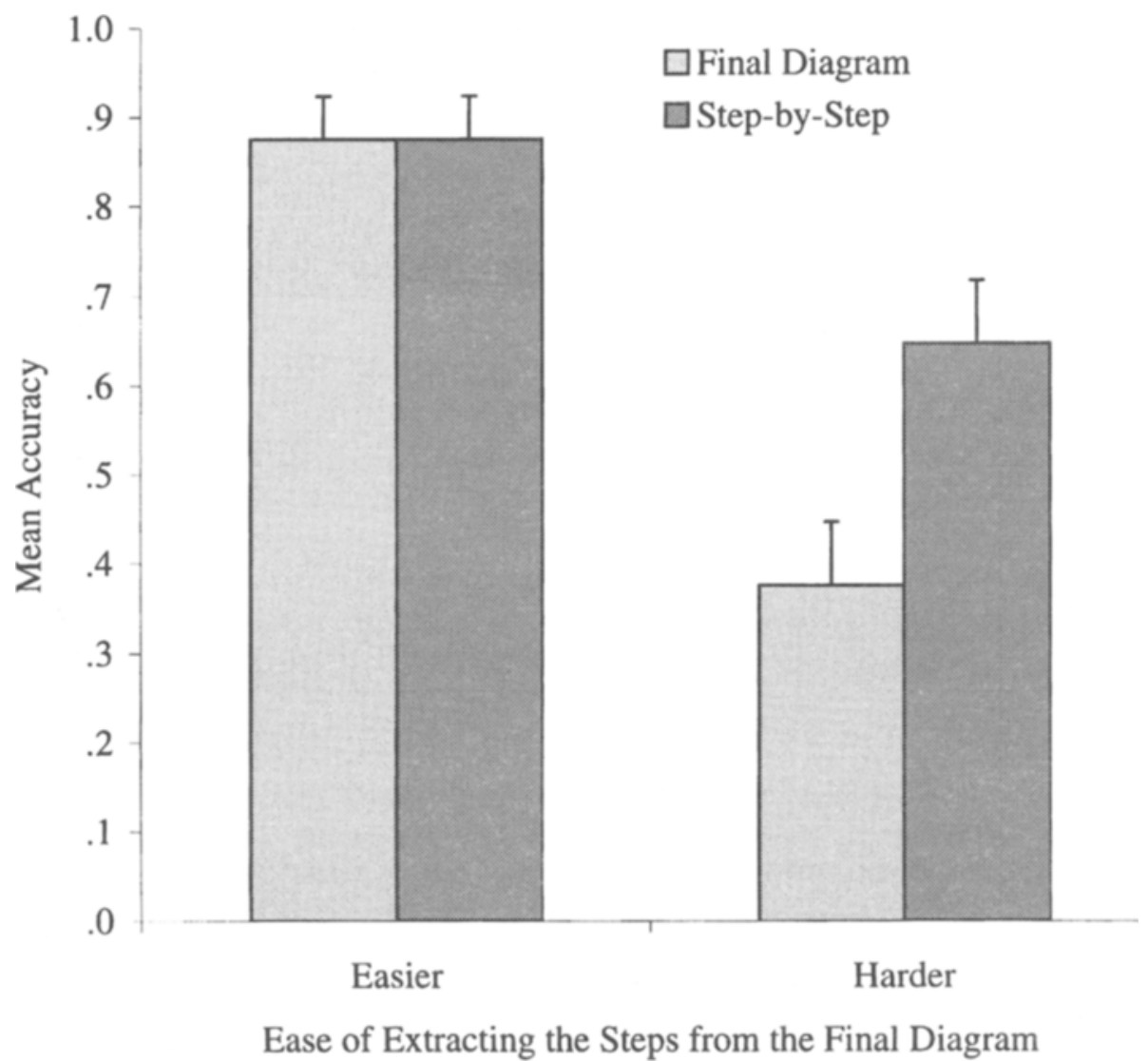

Figure 4. Mean accuracy $(+S E)$ as a function of the ease of seeing the steps in, or inferring the steps from, the final diagram for the final-diagram and step-by-step conditions in Experiment 3 ( $n=24$ per condition). Each bar shows the mean level of accuracy collapsed across the 13- and 16-step objects. 
ease of extracting the steps such that the subjects in the final-diagram condition are comparable to those in the step-by-step condition when it is easier to extract the steps but not when it is harder to extract the steps.

All four predictions were clearly supported. The subjects were much more accurate when it was easier to see or infer the steps in the final diagram $(M=.88)$ than when it was harder $(M=.51)\left[F(1,46)=45.22, M S_{\mathrm{e}}=0.1411\right.$, $p<.001$ ] . Accuracy also decreased from a mean of .81 for the 13-step objects to a mean of .57 for the 16-step objects $\left[F(1,46)=19.28, M S_{\mathrm{e}}=0.1429, p<.001\right]$. Similar to Experiments 1 and 2, the subjects in the stepby-step condition performed marginally better than those in the final-diagram condition, with means of .76 and .63 , respectively $\left[F(1,46)=3.45, M S_{\mathrm{e}}=0.2552\right.$, $p<.07]$. Most importantly, as shown in Figure 4, the predicted interaction between instructional condition and ease of extracting the steps from the final diagram was reliable $\left[F(1,46)=6.24, M S_{\mathrm{e}}=0.1411, p<.02\right]$, with a mean difference between the two conditions of .00 for the easier-to-extract-the-steps objects but .27 for the harder-to-extract-the-steps objects. Regardless of the number of steps, the subjects who had final diagrams performed just as well as the subjects who had step-by-step diagrams when it was easy to see or infer the steps in the final diagrams. But, when it was hard to see or infer the steps, they performed worse.

Unlike in Experiments 1 and 2, number of steps did not interact reliably with instructional condition $[F(1,46)=$ 2.95, $\left.M S_{\mathrm{e}}=0.1429, p>.09\right]$, although the pattern of means mimicked the earlier findings. This finding is consistent with our hypothesis that that interaction in the earlier experiments was largely due to a natural correlation between number of steps and ease of extracting the steps from the finished-object diagram. Finally, number of steps did not interact with ease of extracting the steps $\left[F(1,46)<1, M S_{\mathrm{e}}=0.0994\right]$, nor was there a three-way interaction $\left[F(1,46)=1.31, M S_{\mathrm{e}}=0.0994, p>.25\right]$.

These results support our hypothesis that the ease of seeing the assembly steps in, or inferring them from, the final diagram was primarily responsible for the interaction between number of steps and instructional condition in Experiments 1 and 2. Moreover, the observed pattern of results clearly refutes the alternative view that proposes that subjects simply use the final diagram as a goal state against which to check the accuracy of their completed object and that, therefore, all final-state diagrams are created equal. Instead, the interaction between instructional condition and ease of extracting the steps from the final diagram supports our hypothesis that subjects actively use the final diagram during object assembly to help construct a mental model of the current assembly step. We will explore this account of origami construction in more depth in the General Discussion.

Construction time. Two subjects exceeded the time limit on one object each (the duck and the mushroom). In all other cases, the subjects either attempted all of the steps or decided to stop working within the time allotted.
The time per step scores were submitted to a $2 \times 2 \times 2$ ANOVA with instructional condition as a grouping factor and number of steps and ease of extracting the steps from the final diagram as within-subjects factors. On the basis of the results of Experiment 2, we made two predictions concerning the results of this analysis: (1) stepby-step subjects should be more efficient than finaldiagram subjects, and (2) the subjects should be more efficient with the easier-to-extract-the-steps objects than with the harder-to-extract-the-steps objects.

These predictions were supported. Time per step varied across instructional conditions, with a mean of $0.58 \mathrm{~min}$ per step for the final-diagram condition versus 0.39 min per step for the step-by-step condition $[F(1,45)=$ $\left.10.68, M S_{\mathrm{e}}=0.1775, p<.005\right]$. These means are quite similar to those obtained in Experiment 2 (0.53 and 0.37, respectively), even though the exact objects and the number of steps differed across the two experiments. This suggests that construction efficiency is sensitive to factors that generalize across individual objects. The subjects also were much more efficient when working on easier-to-extract-the-steps objects than on harder-toextract-the-steps objects (means of 0.37 and 0.61 , respectively) $\left[F(1,45)=30.14, M S_{\mathrm{e}}=0.0921, p<.001\right]$. Consistent with the time per step results in Experiment 2, but unlike the results for accuracy, the interaction between instructional condition and ease of extracting the steps from the final diagram was not reliable $[F(1,45)=$ $1.14, p>.29]$.

Number of steps marginally affected construction efficiency, with means of 0.43 and 0.54 for the 13 - and 16 step objects, respectively $\left[F(1,45)=3.56, M S_{\mathrm{e}}=0.1580\right.$, $p<.07]$. There was a reliable interaction between number of steps and ease of extracting the steps from the final diagram, which we did not find for accuracy $[F(1,45)=$ $\left.8.19, M S_{\mathrm{e}}=0.0966, p<.01\right]$. For the 13-step objects, the means were 0.25 and 0.62 , respectively, for the easierand harder-to-extract-the-steps objects. For the 16-step objects, the means were 0.49 and 0.60 , respectively. Number of steps did not interact with instructional condition, nor was the three-way interaction reliable $[F(1,45)<1$, and $F(1,45)=2.75, p>.10$, respectively].

A comparison of the results for accuracy and time per step, in both Experiments 2 and 3, indicates that the two variables are affected by somewhat different factors. We have a consistent, a priori explanation for the accuracy results, which we have discussed to some extent already and will consider more fully in the General Discussion. We have several ideas, after the fact, about why the time per step results are somewhat different. Time per step, but not accuracy, presumably is affected by the number of lines of text required to describe the assembly steps. Time per step may also be affected more than accuracy by the complexity of the steps, with more complex steps requiring more time to understand and execute correctly. These two factors are not completely independent, because more complex manipulations often require more words to describe. These two factors may also be partially 
confounded with number of steps, both in our materials and in origami more generally. Short, simple steps from the materials of Experiment 3 included (1) "turn the figure over from right to left" (shield, mushroom) and (2) "fold the point of the longest triangle-the one on the right-over to the right edge of the rectangle" (duck). For an example of a longer, more complex step, see Procedure 4 in the piano instructions shown in Figure 1.

Adequate tests of these hypotheses will have to wait for future research in which the presumed causal variables are carefully manipulated. In the General Discussion, we will consider alternative methods for manipulating the ease of extracting the steps from the final diagram that should also be helpful in terms of shedding light on the differences between the results for accuracy and efficiency.

\section{GENERAL DISCUSSION}

The use of diagrams for aiding comprehension, by clarifying and recording everyday tasks and events and by facilitating scientific endeavors, has a long history. It is only in recent years, however, that cognitive researchers have started to consider how and why diagrams provide the benefits they do (e.g., Larkin \& Simon, 1987; Winn, 1989). To this end, diagrams have been classified into a variety of different types (e.g., Hegarty, Carpenter, \& Just, 1991), and both structural (e.g., Lohse, Biolsi, Walker, \& Rueter, 1994; Twyman, 1979) and functional (e.g., Cheng, 1996) analyses of diagrams have been proposed. Nevertheless, the immense variety of diagram types and the varied conditions under which they are used has meant that several important avenues of research have not yet been sufficiently explored.

Our goal was to explore the role of diagrams in object assembly. An informal survey of diagrammed instructions in everyday contexts (e.g., building bookcases and bicycles, folding paper objects) suggests that a common assumption is that there should be a diagram to illustrate each major step in the assembly procedure and a diagram showing the completed object. The results of our three experiments, however, indicate that explicitly illustrating the state change at every step is not necessary if the changes are readily inferable from the diagram illustrating the completed object.

\section{Factors That Affect the Accuracy of Origami Construction}

Our subjects folded origami objects in one of three instructional conditions: text only, text plus a diagram showing the completed object (what we refer to as a final diagram), and text plus step-by-step and final diagrams. The most interesting results involved the final-diagram condition. We found that the benefit of a final diagram depended primarily on the ease of extracting the individual assembly steps from that diagram. In Experiments 1 and 2, we took advantage of the natural correlation between the number of assembly steps and the ease of ex- tracting those steps from the final diagram: The more steps there are, the more likely it is that the early steps will be obscured in the final diagram as a result of completing the later steps. Thus, in these two experiments, we found that the final diagrams conferred as much of an advantage (over text alone) for accuracy as step-by-step diagrams when there were few steps $(5-10)$ but no advantage when there were many steps (16-31). In Experiment 3, we manipulated both the number of steps and the ease of seeing those steps in, or inferring them from, the final diagram. As we predicted, the ease of extracting the steps from the final diagram, rather than the number of steps, was the primary causal variable in the interaction with instructional condition.

The fact that the ease of extracting the steps from the final diagram was causally related to construction accuracy for objects containing both 13 and 16 steps increases our confidence in the validity of our results. Nevertheless, it would be better in future research to instantiate this manipulation within, rather than across, objects. For example, the final diagram could show either a frontal or side view of the constructed object. ${ }^{5}$ Alternatively, one could manipulate whether the diagram shows all the internal details (e.g., fold lines) of the object or just the outline shape. These methodologies have the added advantage that they would control extraneous variables in a way that should facilitate investigation of the similarities and differences in the factors that affect construction accuracy and efficiency.

The results of Experiment 3 indicated that the step-bystep diagrams had a somewhat greater advantage over a final diagram for 16-step objects than for 13-step objects, especially when the steps were difficult to extract from the final diagram, but these patterns were not statistically reliable. Thus, over a moderate range, the number of assembly steps does not appear to be crucially important for determining the effectiveness of a final diagram for supporting object assembly. Nevertheless, it seems reasonable to predict that, at some point, the sheer number of steps might well make an independent contribution to construction difficulty. We were limited to no more than 16 steps in Experiment 3, because, beyond 16 steps, we could not find any objects for which it seemed easy to extract the steps from the final diagram. Often, we found that the last half of the steps (approximately) were easy to see in, or infer from, the final diagram, but not the first half of the steps. In these cases, our analysis of this task suggests that a judiciously selected subgoal diagram partway through the assembly process would be especially beneficial.

Another important factor impacting the success of object assembly may be the difficulty or complexity of the steps. More complex folds may be in greater need of diagrammatic support. In the origami domain, it is our impression that the number of steps and the difficulty of the required manipulations are positively correlated: Objects that have more steps also tend to require more complex 
folds. Sorting out the relative importance of these two factors will require some means of assessing step difficulty, which we did not attempt in the present research.

\section{The Role of Diagrams in Object Assembly}

The results of our three experiments support our hypothesis that the role of diagrams in object assembly is to facilitate the processes of (1) constructing a mental model of the current assembly step and (2) mapping the model to the under-construction object. Moreover, the results of Experiment 3 refute an alternative hypothesis that posits that final diagrams are used only to check the accuracy of one's construction attempt once it has been completed. In this section, we summarize our analysis of the cognitive requirements of assembly tasks, and we indjcate how our origami data support this analysis. We also discuss some of the processes and representations we suspect are involved in coordinating the information contained in verbal instructions and a final diagram. However, a complete process model of origami is beyond the scope of this paper.

We posit that object assembly tasks require builders to construct and continually update a mental model of the operations described in the text as they progress through the assembly instructions. At each step, the mental model of the required operation(s) must be mapped onto the actual object under construction, so that the operation(s) represented internally can be carried out on the corresponding part(s) of the object. The ease of executing the mental model construction and model-to-object mapping processes depends, we hypothesize, on the type of instructions available. In particular, it depends critically on the nature of the diagrams available.

When constructing objects guided only by written instructions, people are likely to make errors in both the model construction and mapping processes, due to the increased load on working memory. In contrast, builders who have the benefit of step-by-step diagrams will be less likely to make such errors, because those diagrams facilitate construction of the mental model and simplify the task of managing the correspondence between the mental model and the physical object. Moreover, these builders will be more likely to detect and recover from any errors they do make, because the diagrams provide feedback on construction accuracy at most steps in the construction process. Consistent with this analysis, we found in both Experiments 1 and 2 that construction accuracy was higher in the step-by-step condition than in the text-only condition.

Now consider written instructions that are accompanied by a single diagram showing what the object should look like when constructed correctly. Our task analysis suggests that the effectiveness of a final diagram for supporting object assembly should depend on the ease of seeing the individual assembly steps in, or inferring them from, that diagram. Using Glenberg and Robertson's (1999) terminology, we might talk about the ease of indexing (i.e., referencing) the written instructions to the accompanying diagram. Larkin and Simon (1987) sug- gest, more generally, that a diagram's effectiveness depends on the ease of extracting information from it.

When the assembly steps are easy to extract from the final diagram, builders can construct two sets of links (i.e., mappings) between the information provided to supplement the mental model linking the written instructions to the under-construction object that we suggest is created under all instructional conditions. One mapping connects the text to the final diagram. This is possible because some of the parts and landmarks described in the text are visible in the final diagram. A second mapping connects the physical object to the final diagram due to the visual similarity between them. The text-todiagram and object-to-diagram links, which are available only when the assembly steps are easy to extract from the final diagram, serve as important constraints that facilitate and support the model construction process. These links help builders to (1) check the accuracy of their mental model of the current operation(s) to be executed and (2) correct any inaccuracies discovered. Thus, when the individual assembly steps are easy to extract from the final diagram, that diagram is sufficient to support object assembly, and step-by-step diagrams are largely superfluous.

When it is hard to extract the assembly steps from the final diagram, however, it will be very difficult or impossible for builders to identify links between the final diagram and either the verbally described steps or the object under construction. In these situations, the final diagram does not provide external support for mapping the text onto the under-construction object. Thus, similar to the text-only condition, the mental model of the current assembly step alone will be primarily responsible for guiding the construction process. Moreover, alternative mental models must be considered in working memory, and the appropriate model must be selected without diagrammatic feedback. Due to the increased load this places on working memory, the errors inherent in relying on a continually changing mental model will compound, and construction accuracy will suffer. The interactions between instructional condition and ease of extracting the steps (Experiment 3 ) and number of steps (Experiments 1 and 2) support this analysis.

\section{The Importance of Prior Knowledge}

Given that mental model construction is facilitated when it is easy to extract the individual assembly steps from the final diagram, it is reasonable to ask what makes the steps easy to see in, or infer from, that diagram. Although it seems like this question ought to have a straightforward answer, that turns out not to be the case. In Experiment 3 , we intended to manipulate the ease of extracting the steps from the final diagram for objects having three different numbers of steps $(10,13$, and 16$)$, but our subjects agreed with our classifications for only two of the three pairs of objects (those containing 13 and 16 steps). Thus, the ease of extracting individual assembly steps from a final diagram seems to depend to some 
extent on characteristics of the viewer. We suspect that the expertise of the builder with respect to the domain of application may be important. In origami, with greater expertise likely comes increased knowledge of the effects of different manipulations of the paper, thereby facilitating the process of mentally undoing the manipulations to verify a designated assembly step from the final diagram. We have constructed very many origami objects in the process of selecting stimuli for this research, and, thus, we are considerably more skilled in this domain than are our subjects (who gave themselves a mean origami experience rating slightly above "only a little previous experience").

Mayer and Gallini (1990) found a stronger benefit of their parts-and-steps diagrams for low-prior-knowledge learners than for high-prior-knowledge learners. The interaction in their data was such that high-prior-knowledge learners often performed comparably across instructional conditions, regardless of the types of diagrams presented, whereas low-prior-knowledge learners showed better performance with parts-and-steps diagrams. In the present experiments, we used students who were not highly skilled at constructing origami objects. If expertise in this domain results in an increased ability to infer the individual assembly steps from the final diagram, we might expect less variability in performance across objects in this instructional condition for builders who are highly skilled. We are not aware of any research examining the possible interaction between domain knowledge and type of diagrammatic instructions on success at executing assembly procedures.

\section{Relation to the Findings of Marcus et al. (1996)}

Finally, we would like to consider the relation between our results and those of Marcus et al. (1996). As we noted in the introduction, their study was the only one we found that investigated an object assembly task. In one sense, our results for the final-diagram condition are opposite to the results reported by Marcus et al. They found that the benefit of final diagrams increased monotonically with problem difficulty (operationally defined in terms of the number of connections that had to be considered simultaneously in working memory), whereas we found that the benefit rather dramatically dropped off when problem difficulty increased.

There are several differences between our materials and those of Marcus et al. (1996), however, that may be important to consider. First, the hardest assembly task used by Marcus et al. is probably similar in difficulty to the easiest tasks we used. More importantly, for all of Marcus et al.'s tasks, all the steps were visible in the final diagram. In contrast, our tasks varied widely in the degree to which the individual steps were visible in, or inferable from, the final diagram. Our results strongly indicate that this difference is theoretically important. Diagrams are beneficial when they facilitate mental model construction (e.g., Bauer \& Johnson-Laird, 1993;
Glenberg \& Langston, 1992; Glenberg \& Robertson, 1999), and mental model construction is facilitated when it is easy to extract the individual assembly steps from the final diagram. Problem difficulty is an important factor only if it impacts mental model construction. At a fundamental level, however, we agree with Marcus et al. that what is critical is the amount of information that must be held or manipulated in working memory. When final diagrams are helpful, they reduce the load on working memory.

\section{REFERENCES}

Bauer, M. I., \& JohnSon-Laird, P. N. (1993). How diagrams can improve reasoning. Psychological Science, 4, 372-378.

ChENG. P. C.-H. (1996). Functional roles for the cognitive analysis of diagrams in problem solving. In G. W. Cottrell (Ed.), Proceedings of the Eighteenth Annual Conference of the Cognitive Science Society (pp. 207-212). Mahwah, NJ: Erlbaum.

Froebel-Kan Co. (1991). Origami 12. Torrance, CA: Heian International.

Glenberg, A. M., \& Langston, W. E. (1992). Comprehension of illustrated text: Pictures help to build mental models. Journal of Memory \& Language, 31, 129-151.

GlenberG, A. M., \& RoberTson, D. A. (1999). Indexical understanding of instructions. Discourse Processes, 28, 1-26.

Hegarty, M., Carpenter, P. A., \& Just, M. A. (1991). Diagrams in the comprehension of scientific texts. In R. Barr, M. L. Kamil, P. Mosenthal, \& P. D. Pearson (Eds.), Handbook of reading research (Vol. 2, pp. 641-668). New York: Longman.

Hegarty, M., \& Just, M. A. (1993). Constructing mental models of machines from text and diagrams. Journal of Memory \& Language, 32, 717-742.

KaSahara, K. (1973). Origami made easy. Elmsford, NY: Japan Publications.

LARKIN, J. H., \& Simon, H. A. (1987). Why a diagram is (sometimes) worth ten thousand words. Cognitive Science, 11, 65-99.

LoHSE, G. L., Biolsi, K., WALker, N., \& RUeter, H. H. (1994). A classification of visual representations. Communications of the $A C M$, 37(12), 36-49.

Marcus, N., Cooper, M., \& Sweller, J. (1996). Understanding instructions. Journal of Educational Psvchology, 88, 49-63.

Mayer, R. E., Bove, W., Bryman, A., Mars, R., \& Tapangco, L. (1996). When less is more: Meaningful learning from visual and verbal summaries of science textbook lessons. Journal of Educational Psychology, 88, 64-73.

MAYER, R. E., \& GALLINI, J. K. (1990). When is an illustration worth ten thousand words? Journal of Educational Psychology, 82, 715-726.

MONTROLL, J. (1992). Easy origami. New York: Dover.

Neale, R. \& Hull, T. (1994). Origami, plain and simple. New York: St. Martin's.

SaKADE, F. (1958). Origami: Japanese paper-folding (Book 2). Rutland, VT: Tuttle.

Teмко, F. (1986). Paper pandas and jumping frogs. San Francisco: China Books.

Twyman, M. (1979). A schema for the study of graphic language (tutorial paper). In P. A. Kolers, M. E. Wrolstad, \& H. Bouma (Eds.), Processing of visible language (Vol. 1, pp. 117-150). New York: Plenum.

WINN, W. (1989). The design and use of instructional graphics. In H. Mandl \& J. R. Levin (Eds.), Knowledge acqui ition from text and pictures (pp. 125-144). Amsterdam: Elsevier.

\section{NOTES}

1. We should note that no prior domain knowledge was needed to interpret the diagrams and make the connections, due to the simplicity of 
the problems. In fact, there was no real need for the subjects to understand the circuits they were constructing. Thus, although the results of this research further our knowledge of object assembly, they probably do not tell us much about the specific domain of circuit design

2. Because Experiment 1 was conducted as a class project under extreme time pressure, it was not possible to assess interrater reliability. However, the results of Experiment 2 replicate those of Experiment 1, thereby serving equally well to validate the findings.

3. What we mean here is that all the final diagrams represent the completed object equally well. Although this would not be the case if some final diagrams were of poor quality perceptually or showed the completed object from an unusual angle, these factors are not an issue with our materials. As far as we can tell, all of our final diagrams are good depictions of the completed objects. They are of comparable visual quality, and all show the completed object from the perspective in which the subject's constructed object would appear at the conclusion of the final step in the written instructions (assuming that the object was constructed correctly, of course).

4. Here, we would simply like to note that it is perhaps not too surprising that our predictions concerning the ease of extracting the steps from the final diagrams were not all borne out. Our predictions were made after we had studied the step-by-step diagrams, because such diagrams normally appear alongside the text (or sometimes instead of text) in origami books. Thus, our perspective on the final diagrams was colored by our prior knowledge of the step-by-step diagrams, which our manipulation-check (and final-diagram condition) subjects did not see. It is possible that this knowledge could have altered our understanding of some of the final diagrams.

5. We thank Art Glenberg for suggesting this manipulation.

(Manuscript received March 23, 1999;

revision accepted for publication December 7, 1999.) 